\title{
Buscando Orientaciones: Pautas para la Enseñanza de la Ética Profesional en Psicología en un Contexto con Impronta Postmoderna
}

\section{Searching for Orientation: Guidelines for Teaching Professional Ethics in Psychology in a Context with a Post-Modern Emphasis}

\author{
Diana Pasmanik y María Inés Winkler \\ Universidad de Santiago de Chile, USACH
}

\begin{abstract}
Resultados de estudios realizados en Chile sugieren que los psicólogos prefieren tomar decisiones de orden ético sobre la base de su juicio personal y su experiencia profesional, valorizando menos la utilidad del código de ética profesional (Alvear, Pasmanik, Winkler \& Olivares, 2008). Partiendo de este antecedente, se recabó información para situar la enseñanza de la ética profesional en las transformaciones que actualmente experimenta la educación superior y se buscó elementos de juicio para la toma de decisiones con respecto a la enseñanza de la ética en el pregrado. En este artículo se presentan y discuten algunos acercamientos a dicha formación que se aplican en la enseñanza de la psicología y se proponen algunas orientaciones para decidir qué, cuándo y cómo enseñar ética en psicología.
\end{abstract}

Palabras clave: ética, principios éticos, identidad profesional, normas, enseñanza de la psicología.

\begin{abstract}
Previous survey's results suggest that Chilean psychologists prefer to make ethical decisions based on their personal judgment and professional experience, and place less value on the utilization of a Professional Code of Ethics (Alvear, Pasmanik, Winkler, \& Olivares, 2008). With this background, information was obtained in order to position the teaching of professional ethics in the transformation that is currently being experienced in higher education, and criteria for decision making on teaching ethics at the undergraduate level were also sought. Some of the approaches to ethics education in psychology are presented and discussed, and guidelines are proposed for deciding what, when and how to teach ethics in psychology.
\end{abstract}

Keywords: ethics, ethics principles, professional identity, norms, teaching of psychology.

\section{Introducción}

Resultados de un estudio realizado en Chile y recientemente publicado (Alvear, Pasmanik, Winkler \& Olivares, 2008) sugieren que habría una postura crítica de parte de las y los psicólogos ${ }^{1}$ chilenos con respecto al Código de Ética del Colegio de Psicólogos de Chile. De un total de 170 psicólogos, de los cuales el 81,1\% manifestó conocer el código de ética, un $60,4 \%$ se manifestó "con- trario" a los códigos de ética profesional, valorando otros recursos para enfrentar problemas éticos, tales como la experiencia personal $(53,3 \%)$ y el dominio de un buen manejo técnico (55\%) conviniendo, además, en que quien ejerce de modo serio y responsable la profesión no requeriría recurrir al Código de Ética (56,2\%).

Una explicación, al menos parcial, de esta situación puede estar en la influencia de la postmodernidad en nuestra sociedad

Diana Pasmanik, Escuela de Psicología, Universidad de Santiago de Chile, USACH, Santiago, Chile.

María Inés Winkler, Escuela de Psicología, Universidad de Santiago de Chile,USACH, Santiago, Chile.

La correspondencia relativa a este artículo debe ser dirigida a Diana Pasmanik, Escuela de Psicología, Universidad de Santiago de Chile, Avda. Ecuador 3650, $3^{\text {er }}$ piso, Estación Central, Santiago, Chile. E-mail: diana.pasmanik@usach.cl Este trabajo se realizó gracias al apoyo de la Comisión Nacional de Investigación Científica y Tecnológica de Chile (CONICYT, proyecto FONDECYT N ${ }^{\circ}$ 1080484) y del Departamento de Investigación Científica y Tecnológica (DICYT), Universidad de Santiago de Chile (USACH).

Asumimos la importancia de la distinción lingüística de género; no obstante, para facilitar la lectura del texto, en adelante esta será obviada. 
$\mathrm{y}$, particularmente, entre sus individuos más letrados. Desde mediados del siglo XX, se reconoce a la postmodernidad como un nuevo período en el devenir de nuestra civilización. Surge junto al desencanto con los meta-relatos, es decir, las grandes ideologías o sistemas de pensamiento que aparecieron con la Ilustración. Estas orientaban a la sociedad con las ideas que el progreso y el desarrollo social tienen un fin, que existe una verdad única y absoluta y que el desarrollo humano depende fundamentalmente de la razón (Bauman, 2004; Díaz, 1999; Lipovetsky, 1998; Vattimo, 2004).

Sin embargo, es posible atribuir estos resultados a otros factores que también pueden estar influyendo, tales como la necesidad de revisar -y eventualmente actualizarel Código de Ética Profesional del Colegio de Psicólogos de Chile, cuya última versión data de 1999. Cabe considerar, además, las atribuciones limitadas que aún tiene el Colegio de Psicólogos para ejercer la tuición ética de la profesión en Chile.

Un factor relevante de la postura crítica es la formación en ética recibida durante los años de formación universitaria. Algunas investigaciones y tesis para la obtención del título profesional realizadas en Chile en décadas anteriores así lo sugieren (Blanco \& Ite, 1994; Morales, Sziklai, Díaz \& Scharager, 1988). A lo largo de los años, una crítica recurrente es la formación escasa, teórica y descontextualizada del ejercicio profesional. Al mismo tiempo, se aprecia una demanda por una enseñanza de la ética que privilegie la reflexión y el debate, sobre la base del análisis de casos.

Estos datos constituyen nuestro punto de partida para la búsqueda de pautas para la formación en ética profesional en psicología en Chile. No pretendemos culminar con una propuesta acabada, asunto que, por lo demás, tampoco parece estar zanjado en otras latitudes (De las Fuentes, Willmuth \& Yarrow, 2005; Falender, 2006, Agosto). Nuestra intención, más bien, es proponer un conjunto de orientaciones que considerar al momento de planificar la formación.

Iniciaremos nuestra exposición revisando algunos antecedentes que permiten situar la enseñanza de la ética profesional en las transformaciones que está experi- mentando la educación superior en la actualidad. Después, entregaremos algunos elementos de juicio para la toma de decisiones con respecto a la formación en ética profesional, para, ulteriormente, presentar y discutir algunos acercamientos a dicha formación que se aplican en la enseñanza de la psicología. Culminaremos con la propuesta de algunas orientaciones para la enseñanza de la ética profesional. Si bien nuestra reflexión se realiza teniendo presente la formación específica de los psicólogos, la mayor parte de la información recabada y su análisis, así como las orientaciones propuestas, se ajustan a la formación profesional en general.

\section{La Presencia de la Ética en la Formación Universitaria a Inicios del Siglo XXI}

En la primera década del siglo XXI, el contexto de mayor generalidad de la formación profesional lo constituyen la globalización y la instalación de una sociedad del conocimiento y digitalizada. Diversas iniciativas que llevan a la unificación de países en formas mayores de organización, tales como la Comunidad Europea y el MERCOSUR, requieren de profesionales preparados para insertarse en distintos escenarios y adaptarse a culturas diferentes. En este contexto cobra fuerza la opción por una formación profesional basada en competencias que, en Europa, da origen al proyecto Tuning (González \& Wagenaar, 2003). Ideado para facilitar una formación profesional más homogénea al interior de la Comunidad Europea, por su intermedio se espera establecer la equivalencia de las distintas titulaciones en el continente.

La formación basada en competencias permite establecer un lenguaje común que se refleja en la formulación de perfiles profesionales y moldea los diseños curriculares y las estrategias de formación.

En el proyecto Tuning (González \& Wagenaar, 2003) se introduce la ética como una competencia transversal interpersonal, bajo la denominación de compromiso ético. De este modo, se otorga un nuevo impulso a la formación en ética en la universidad (Bolívar, 2005; Boni \& Lozano, 2005). Este constituye una invitación a superar la neu- 
tralidad que caracterizó la formación liberal, vinculada a la Ilustración, y su creencia relativa a que el solo conocimiento bastaba para que los individuos fueran mejores. También era una invitación a superar el adoctrinamiento ideológico de otros momentos, subrayándose el componente moral inherente a la educación (Bolívar, 2005). Sin embargo, la inserción de la ética se produce sobre la base de un enunciado ambiguo. Puede ser entendida tanto como atender -en la formación- a la dimensión moral de la personalidad, comprendiendo las competencias éticas como profesional y como ciudadano, o bien en el sentido restringido del aprendizaje de un código deontológico (Bolívar, 2005). Este último consiste en el conjunto de principios y normas que orientan la actuación profesional, de acuerdo a los deberes y obligaciones de la profesión.

\section{La Formación Universitaria en el Dominio Ético-Moral}

$\mathrm{Al}$ referirnos al dominio ético-moral, estamos empleando dos conceptos que requieren de definición. Orellana Benado (1994) define la ética como una disciplina filosófica orientada a la reflexión acerca de las costumbres, tarea que cumplirían, “... desde el punto de vista de los valores, los principios y las normas en cuyos términos se pretende responder a la pregunta acerca de cómo, en principio, debe vivirse la vida" (p. 41). Para Bolívar (2005), la ética es una reflexión crítica de segundo orden acerca de la experiencia moral humana y prescribe los modos de comportamiento justificables. En su origen etimológico ética deriva de la palabra griega ethos, "morada", "lugar donde se vive", y también significa "carácter" y "conciencia". Alude, por lo mismo, a la forma como conducirse entre el nacimiento y la muerte (Holzapfel, 2000). En la actualidad este "habitar" resulta altamente complejo, debido a que la sociedad no alcanza a modificar sus hábitos y costumbres con la misma rapidez con la que se producen los cambios tecnológicos y el conocimiento. Cuando los cambios se producen, no se alcanzan a arraigar cuando ya quedan obsoletos (Bilbeny, 1997). Por este hecho, hoy en día se hace imperativa la autonomía moral, en términos piagetanos. La ética, como lo sugiere su calidad de "conciencia", demanda de reflexión, que sea crítica, de segundo orden y acerca de valores y comportamientos, dirimiendo con respecto a aquellos que resultan justificables.

Moral tiene su origen en mores, costumbres (Bilbeny, 1997; Montero, 2004). Se refiere a los hábitos y costumbres que regulan la propia actuación y la convivencia social.

Así, el dominio ético-moral comprende los hábitos y costumbres de un grupo humano y su apropiación y la reflexión crítica acerca de estos mismos, de su origen, de los valores que les dan soporte y de la idoneidad de las actuaciones y comportamientos propios y ajenos.

El concepto de compromiso ético, en su ambigüedad, refleja la diversidad de la formación en el dominio ético-moral en los espacios universitarios en la actualidad, en los que coexisten distintos acercamientos, con supuestos de base y fines diferentes. $\mathrm{Su}$ análisis permite concluir que estos no son excluyentes entre sí sino, por el contrario, podrían combinarse, con diferentes énfasis, a lo largo del trayecto de la formación universitaria. Como veremos, algunos acercamientos tienen un carácter transversal a la formación, apuntando al estudiante en cuanto persona, ciudadano y actor social; otros se refieren a modos de concebir y enseñar la ética profesional.

\section{La Formación Ético-Moral de Carácter Transversal}

Los acercamientos a la formación con acento transversal y mayor presencia parecen ser la formación para la ciudadanía y la responsabilidad social universitaria. La formación para la ciudadanía se implementa no solamente en el ciclo terciario sino, también, en el medio escolar. En el marco universitario se fundamenta en la responsabilidad del profesional con su sociedad, como ciudadano e integralmente como persona (Martínez, Buxarrais \& Bara, 2002). Se vincula con la educación de la moral cívica de una sociedad democrática, propuesta por Cortina (1995), basada en lo que la autora denomina la ética de los mínimos, que asegura la intención por promover la justicia, sustentada en los valores de libertad, 
igualdad y solidaridad y en los derechos humanos.

En la formación universitaria se aprecia, también, una orientación a la promoción de valores y actitudes prosociales, a través del desarrollo de programas elaborados en el marco de la noción de responsabilidad social universitaria, es decir, aquella que le corresponde a las universidades con respecto a la comunidad en que están insertas y al país en general, aportando en la solución de problemas en beneficio de la sociedad (Arratia Figueroa, 2008; Martínez de Carrasquero, Mavárez, Ligibther \& Carvallo, 2008).

Por otra parte, están los esfuerzos por desarrollar procedimientos para la promoción en la formación universitaria del compromiso ético como competencia genérica, mediante un diseño curricular basado en competencias, en el que se destaca, en el concierto iberoamericano, la Universidad de Deusto (Villa \& Poblete, 2007).

\section{La Formación en Ética Profesional}

Otra vertiente, que no se contradice con la anterior sino, por el contrario, la complementa, es la formación orientada a la ética profesional. Las profesiones se caracterizan por ser ocupaciones que cumplen una función de alta relevancia social, razón por la cual requieren de una preparación rigurosa. La complejidad y especificidad del conocimiento que los profesionales manejan determina un alto grado de poder y autonomía en su desempeño (Gyarmati, 1984). Mediante el ejercicio profesional, es posible tanto beneficiar como perjudicar al prójimo, sea este un individuo, grupo, familia, organización o comunidad. De ello deriva la importancia de la ética profesional, mediante la cual se promueven las buenas prácticas profesionales y se previene el perjuicio que el ejercicio profesional negligente o equivocado pudiera ocasionar.

En los últimos años, la noción tradicional de las profesiones como ocupaciones de ejercicio liberal y la idea que la obtención de un título profesional permite predecir un cierto desarrollo de carrera, se ven vulneradas por la diversificación de la ciencia en áreas y subdisciplinas y de las profesiones en áreas de especialización, así como por el surgimiento, en términos disciplinarios, de campos de frontera. En este contexto, la existencia de principios profesionales éticos sería más importante hoy en día que en épocas anteriores, debido a los cambios que experimentan las profesiones y los desajustes que ello ocasiona en la identidad profesional (Hirsch Adler, 2003).

La dificultad para orientar la actuación profesional en su marco ético sería mayor debido a que los profesionales deben aplicar la deontología profesional en contextos y situaciones para los cuales esta no fue pensada. La conducción del análisis de las situaciones éticamente complejas en base a los principios de la profesión podría facilitar la toma de decisiones cuando la norma no baste para encontrar una respuesta.

Este es el marco de referencia en el cual orientaremos la reflexión a tres parámetros que habría que considerar en toda planificación curricular y de los cuales la enseñanza de la ética profesional no estaría exenta: ¿Qué contenidos enseñar? ¿Cuándo enseñarlos? ¿Cómo enseñarlos? Nos ocuparemos de las dos primeras interrogantes en el próximo apartado. En esta ocasión, optamos por hacer notar algunas particularidades de la ética que condicionan su enseñanza, más que hacer un listado de temas relevantes. Un contenido fundamental lo constituyen los códigos de ética. Los trataremos, pero poniendo el énfasis en las demandas de su uso, en las que reside la dificultad -y también el desafíopara la formación. Después, nos referiremos a la identidad profesional y a sus alcances para la formación en ética. En el apartado subsiguiente describiremos algunas aproximaciones vigentes para la formación en ética profesional en psicología, según las opciones identificadas en la literatura especializada.

\section{Formación en Ética Profesional: Qué Enseñar y Cuándo Hacerlo}

\section{Los Códigos de Ética Profesional}

La enseñanza de la ética profesional incluye necesariamente como contenido el Código de Ética Profesional. Los códigos de ética en psicología están constituidos por normas -generales, que cumplen una 
función educativa, y específicas, que son las normas deontológicas propiamente tales- y un conjunto de principios, es decir, normas o ideas fundamentales que rigen el pensamiento o la conducta (Real Academia Española, 2001). Los principios poseen un carácter aspiracional, vale decir, son ideales a alcanzar y, por lo mismo, no tienen carácter normativo (Código de Ética Profesional, Colegio de Psicólogos de Chile, 1999; Declaración Universal de Principios Éticos para Psicólogas y Psicólogos, Sociedad Interamericana de Psicología [SIP], 2008).

Estos códigos tienen por propósito regular qué es lícito y qué no en el ejercicio profesional. Hirsch Adler (2003) señala que permiten determinar la licitud, en base a criterios y principios, en situaciones dilemáticas, es decir, aquellas en que dos o más principios están en conflicto y es necesario decidir cuál cumplir. Además, los códigos declaran los principios y valores y los criterios específicos de la profesión, desde la perspectiva de sus miembros. Poseen, al mismo tiempo, una función informativa hacia clientes, usuarios, poderes públicos, otros profesionales y la sociedad en general y cumplen una función protectora de la profesión en la defensa de la competencia profesional, la conducta correcta y la defensa de los intereses profesionales.

Los códigos de ética se constituyen, por lo tanto, en un instrumento informativo que guía la actuación profesional, pero las decisiones relativas a cómo actuar requieren de la reflexión y el juicio del profesional. Ilustra esto la presentación del Código de Ética del Colegio de Psicólogos de Chile (1999), que culmina con la advertencia:

Un Código de Ética profesional es un conjunto de normas de conducta profesional respaldadas por principios que constituyen su marco teórico-ético. Necesariamente tiene un carácter no exhaustivo, no pudiendo abarcar todas las conductas y sus matices y deja un margen razonable de interpretación. (p. 4)

Por otra parte, la enseñanza de la ética demanda, a su vez, la enseñanza de las leyes reguladoras del ejercicio profesional. Nuevamente aquí no basta con conocer las leyes sino, también, habrá que dirimir lo que en ocasiones resulta difícil cuando las leyes y la ética profesional entran en conflicto. Este problema ha sido estudiado por Pope y Bajt (1995), quienes mostraron que psicólogos caracterizados por una gran experticia ética reconocían haber faltado a la ley en asuntos de carácter profesional, por razones de conciencia. La publicación de sus resultados generó en su momento una fuerte controversia, con argumentaciones a favor y en contra (Ansell \& Ross, 1995; Kalichman, 1995; Van Eenwyk; 1995). Más allá de ello, pone en discusión el carácter controvertido de la dimensión ética en el ejercicio profesional de la psicología, que se refleja cuando los valores están en pugna o bien frente a situaciones dilemáticas.

\section{La Identidad Profesional}

Un elemento que se repite en el análisis de algunos autores con respecto al valor de la ética profesional es la identidad profesional. La identidad es intersubjetiva (depende de las relaciones dialógicas con los demás) y contextual, pues se construye sobre la base del material simbólico que sus comunidades de pertenencia le proporcionan al individuo (Hirsch Adler, 2003).

Más allá de la ética profesional, la profesión en sí misma es un elemento identitario; uno es profesional, lo que indica que ha incorporado el espíritu universitario. La medida en que ello se logre depende de circunstancias personales, pero especialmente del grado de inserción, sensibilidad y apertura para la participación en la vida universitaria (Hirsch Adler, 2003).

Pero el ser profesional también supone ser miembro de una profesión determinada e, incluso, de una de sus especialidades. Esta cualidad identitaria tiene que ser considerada en la formación profesional. Como enfatizan González Maura y González Tirados (2008), al referirse a la formación profesional y aludiendo al modelo de Delors (1996) sobre las áreas del saber, no basta con conocer y saber hacer sino, también, hay que ser profesional.

Para algunos autores, la ética profesional forma parte de la identidad profesional. Hirsch Adler (2003) lo señala refiriéndose a las profesiones en general, mientras que 
Handelsman, Gottlieb y Knapp (2005) lo plantean con respecto a la psicología. En Chile encontramos un ejemplo ilustrativo en el Código de Ética de la Orden que, en su presentación, se plantea que en el mandato del Colegio para su creación “... se enfatiza la ética como valor central de la profesión y su ejercicio" (Colegio de Psicólogos de Chile, 1999, p. 4).

Un ejemplo adicional lo constituye la Declaración Universal de Principios Éticos para Psicólogas y Psicólogos. Este documento fue elaborado mediante la revisión comparada de los Códigos de Ética de psicología de diferentes latitudes del planeta y presentado en el Congreso Internacional de Psicología de Berlín, en Julio de 2008, donde fue ratificada y adoptada formalmente por la International Union of Psychological Science (IUPsyS) y la International Association of Applied Psychology (IAAP). También fue ratificada por la SIP en Diciembre de 2008 (SIP, 2008). El documento comprende cuatro principios: Respeto por la Dignidad de las Personas y de los Pueblos, Cuidado Competente del Bienestar de Otros, Integridad y Responsabilidades Profesionales y Científicas con la Sociedad.

En la primera década del siglo XXI encontramos, entonces, a la psicología buscando aunar criterios para disponer de un marco de referencia único para su ética. Que esto ocurra a través de la declaración de un conjunto de principios y no de un conjunto de normas, como podría ser, por ejemplo, un decálogo, sería consecuencia de las características propias de la época que nos toca vivir. La autonomía moral -un imperativo en la actualidad-con su consecuente énfasis en la responsabilidad personal, demanda de una ética que descanse en el juicio personal, producto de la reflexión y el diálogo que, en definitiva, es un encuentro con el otro.

Para concluir, el ser profesional se constituye en un elemento identitario en dos dimensiones: el ser profesional, es decir, alguien que estudió una carrera que lo legitima y también lo obliga, con derechos y deberes particulares con respecto a la sociedad; y el ser profesional de una profesión específica, como es la psicología. En uno u otro caso la dimensión ética está presente, por lo que los logros de aprendizaje en este dominio forman parte del devenir de una nueva forma de ser. Así, en la tarea de formar en ética profesional habría que considerar al estudiante integralmente. Al mismo tiempo, la formación en ética profesional sería un proceso que se prolongaría a lo largo del plan de estudios durante toda la vida profesional. Este demanda determinar qué enfatizar, según el momento del trayecto de la formación, lo que nuevamente nos remite al cómo enseñar. En nuestro caso, nos limitaremos al ámbito universitario.

\section{Aproximaciones y Modelos para la Enseñanza de la Ética Profesional en Psicología}

Actualmente coexisten distintas formas de enseñar ética profesional en psicología, a las que nos referiremos como aproximaciones y modelos. La distinción entre ambos conceptos la realizamos sobre la base del nivel de especificidad de la propuesta pedagógica: mientras que por aproximación se entenderá un marco general, un modelo involucra diversos componentes cuya articulación es planteada explícitamente por sus autores.

La revisión de la literatura especializada muestra a estas opciones circunscritas a la enseñanza de la psicología clínica y, si bien algunas detallan contenidos, metodologías de trabajo y actividades concretas, las afirmaciones acerca de sus bondades, en su gran mayoría, no aparecen respaldadas empíricamente.

La forma menos estructurada de todos los modos de enseñar ética en psicología es el aprendizaje incidental, aquel que ocurre cotidianamente y que en psicología ocurriría en los espacios de supervisión clínica. Respondiendo a las necesidades de los supervisados y de la situación, pueden transformarse en aprendizajes significativos debido a la pertinencia de su ocurrencia. Como forma de aprendizaje intencionado y sistemático es criticada por Handelsman (1995). Su falta de planificación, y eventualmente de rigor, como intervención educativa no permite asegurar una formación de calidad. 
Aproximaciones Focalizadas en la Enseñanza de la Ética Profesional en Psicología

Nos referimos brevemente en este apartado a dos formas de enseñar ética profesional, reconocidas en psicología. Estas son la combinación de filosofía y el código de ética profesional y el entrenamiento en resolución de problemas. Las calificamos como focalizadas porque abarcan un ámbito específico de la formación. La primera se caracteriza por la combinación de psicología y filosofía (Fine \& Lawrence, 1995), a veces incorporando el marco deontológico y legal de la profesión (Calo, 2000). La segunda trata del entrenamiento de los estudiantes en algún modelo de toma de decisiones éticas. Un ejemplo es el procedimiento de Ajzen (1996), en el que los pasos a seguir son: (a) obtener información para identificar los temas éticos de una situación; (b) identificar a los participantes para determinar qué intereses están en riesgo; (c) generar diferentes alternativas de solución; (d) realizar un análisis de costo y beneficio para cada solución posible y (e) elegir una alternativa y evaluar sus resultados.

$\mathrm{Si}$ bien estas propuestas incorporan aspectos de indiscutible relevancia para la formación en ética profesional, ambas excluyen aspectos importantes de tener presentes en un proceso formativo. El énfasis en la primera se ubica en un conocimiento de tipo fáctico, declarativo. Su fortaleza está en que permite ser aprovechado en la estimulación de la reflexión y del análisis crítico; sin embargo, su debilidad reside también ahí si los esfuerzos por formar en ética profesional se acotan, omitiendo otras áreas cuyo trabajo es igualmente necesario. En la segunda alternativa el conocimiento es procedimental. Tiene la ventaja de integrar el aprendizaje de los contenidos de orden fáctico, requisito para la toma de decisiones, con el análisis de casos concretos, y facilita que los estudiantes experimenten la incertidumbre que supone tomar una decisión en situaciones que, en última instancia, dependen del propio juicio y cuyos alcances suponen una responsabilidad personal. Se corre el riesgo, sin embargo, de omitir aspectos personales del estudiante, que incidirán en la elabo- ración de su juicio y en su comportamiento concomitante.

\section{Modelos Multidimensionales para la Enseñanza de la Ética Profesional en Psicología}

Presentamos aquí tres modelos adicionales, la Formación en Ética Profesional Fundamentada en la Teoría del Desarrollo Moral de Kohlberg; como Competencia Profesional; y como Proceso de Aculturación. Estas resultan en modelos que abarcan en forma más integral la formación en ética profesional. La teoría de Kohlberg (1981) enfatiza el desarrollo del juicio moral. Siguiendo a Piaget, Kohlberg describe el desarrollo del juicio moral organizándolo en tres niveles, preconvencional, convencional y postconvencional, cada uno compuesto de dos estadios. Su teoría, si bien aceptada, ha recibido críticas, una de las cuales alude al hecho que el solo juicio no asegura un comportamiento concomitante.

Recogiendo esta debilidad, Rest (1983), discípulo de Kohlberg, propone un modelo de cuatro componentes que determinarían el comportamiento moral: la sensibilidad moral, la motivación moral, el juicio moral y el carácter moral.

La sensibilidad implica la preocupación por el otro y por anticiparse a los posibles efectos perniciosos que una situación o hecho determinado pueda significarle. Requiere de la capacidad para interpretar una determinada situación como moralmente compleja. El desarrollo de este componente se facilita mediante la promoción de todo aquello que involucre la sensibilidad a la perspectiva del otro. El juicio moral demanda la actividad cognitiva que permite comprender los problemas morales, evaluarlos y escoger los posibles cursos de acción. La motivación moral se refiere a priorizar los valores morales por sobre otros motivos de orden personal. El carácter moral exige el desarrollo de fuerza de voluntad, perseverancia y otras características de orden personal que permitan enfrentar y resolver problemas del dominio moral, sobreponiéndose a las eventuales presiones, amenazas y otras características adversas que sea necesario enfrentar para concretar el actuar correcto. 
Este modelo ha sido empleado en programas de formación de distintas profesiones (Rest \& Narváez, 1994), incluyendo la psicología (De las Fuentes et al., 2005). Existe evidencia empírica que fundamenta estas intervenciones, en que estudiantes han sido evaluados antes y después mediante el $D e$ fining Issues Test de Rest (1979), medición con frecuencia acompañada de algún instrumento o recurso para pesquisar los cambios que pudieran haberse producido en la sensibilidad moral.

Falender (2006, Agosto) se fundamenta en el enfoque basado en competencias para proponer algunas orientaciones para el tratamiento de la ética profesional en el desempeño de la práctica clínica. Ajustándose a la generalidad de los diseños curriculares basados en competencias, distingue tres componentes: el conocimiento (los códigos de ética profesional, las normas, regulaciones y casos legales ejemplares), las habilidades (para reconocer asuntos legales y éticos, para aplicar conocimientos y destrezas en la práctica, para reconocer y reconciliar conflictos entre códigos y leyes relevantes, entre otros) y los valores éticos y actitudes (adoptar un modelo de toma de decisiones ética o bien adaptar el propio, y aplicarlo con integridad personal, auto-conciencia, auto-evaluación y sensibilidad hacia al contexto). La autora también plantea la necesidad de contemplar en la formación la revisión de las decisiones éticas de carácter intuitivo y la importancia de tener presente -y mostrar a los estudiantes- la complejidad inherente tanto a la práctica como a la resolución de problemas de orden ético.

Para cumplir con los propósitos formativos, Falender (2006, Agosto) sugiere el empleo de una diversidad de métodos, dependiendo del tipo de contenido, y concentra parte de su preocupación en la necesidad de elaborar una instrumentación adecuada para la autoevaluación por parte del alumnado y de su seguimiento por parte del docente.

Handelsman et al. (2005) se cuestionan cómo, en su formación como psicólogos, los estudiantes desarrollan el sentido de sí mismos como profesionales éticos. Partiendo del supuesto que la psicología, como profesión y como disciplina científica, constituye una cultura, proponen que la formación en ética profesional puede ser comprendida como un proceso de aculturación. Para explicarlo, se guían por el modelo de aculturación de Berry (1980, 2003; Berry \& Sam, 1997, citados en Handelsman et al., 2005), que comprende dos dimensiones, mantenimiento de la cultura y contacto y participación, y conducen a cuatro posibles estrategias de adaptación: integración, asimilación, separación y marginalización.

La integración supone la capacidad para adoptar los valores éticos de la psicología, conciliándolos con la ética personal de origen. La asimilación se produce cuando los nuevos valores son asumidos y los originarios descartados. Encierra el peligro de una asunción superficial de la ética profesional, focalizada en las normas y no en los principios que las sustentan. En la separación la persona opta por mantenerse en el marco de su ética personal de origen y desde ahí regular su desempeño profesional. Los diversos riesgos de esta estrategia pueden subsumirse en las consecuencias de salirse de los márgenes del ejercicio profesional. La marginalización implica una baja identificación en términos éticos, tanto con la cultura de origen como con la psicología. El extremo de esta estrategia de adaptación la refleja la conducta psicopática en el marco del ejercicio profesional. Estas estrategias, así como las tensiones entre mantenimiento de la cultura y contacto y participación, permitirían diagnosticar el proceso que experimentan los estudiantes en su apropiación de la cultura de la profesión y de su ética en particular y, asimismo, orientar la formación.

Aunque en este modelo la disciplina psicológica es presentada en un sentido general, los ejemplos que los autores proporcionan son de corte clínico como, por ejemplo, el significado de prestarle dinero a un amigo versus prestárselo a un paciente. Cabe señalar que Handelsman et al. (2005) se refieren a la formación de estudiantes que ingresan a lo que en Chile se considera un ciclo de formación profesional (se refieren a new graduate students, p. 62) y que en los Estados Unidos corresponde a programas de magíster y doctorado.

El trabajo docente en este enfoque de- 
manda de una disposición comprensiva hacia al estudiante, orientada a facilitar la revisión de la propia historia personal en materias ético-morales, delineando para sí mismo su cultura ética originaria, mostrarle la nueva cultura -la psicología- y ayudarle en la integración de ambas. Se recomienda que los propios docentes hagan un examen de sí mismos con respecto a su proceso personal de aculturación a la psicología, por lo que tácitamente se transforma en un requisito para implementar una metodología basada en este enfoque. Para tal efecto, Handelsman et al. (2005) formulan algunas sugerencias, tales como la aplicación del genograma ético y la redacción de autobiografías éticas.

Este enfoque puede considerarse una alternativa de respuesta a la necesidad de incluir en la formación la historia personal y, particularmente, el "bagaje" ético-moral con el que se ingresa a la formación. La concepción de la psicología como una cultura, sin embargo, requiere de una mayor elaboración. La relación entre ética y técnica en el psicoanálisis, por ejemplo, no es la misma que en la orientación cognitivo-conductual, lo que supondrá matices distintos en la enseñanza de lo que es ético en la psicoterapia en uno y otro caso. La heterogeneidad de la disciplina también encuentra expresión en sus distintos ámbitos de especialización, lo que es necesario tener en cuenta. Pensamos que el símil más adecuado es el de una cultura que comprende subculturas.

\section{La Ética Positiva como Enfoque de Aproximación a la Formación}

De reciente desarrollo, la ética positiva se denomina así porque supone una perspectiva positiva, no legalista ni punitiva, para concebirla. Knapp y VandeCreek (2006) distinguen entre ética remedial y positiva. La primera se centra casi exclusivamente en los medios (leyes, códigos de ética, orientaciones éticas) y tiene por propósito evitar que los psicólogos puedan dañar a otros mediante su ejercicio profesional. La ética positiva, en cambio, se orienta a la excelencia en el ejercicio de la profesión, sobre la base de valores y principios éticos profundamente arraigados. No se trata, entonces, de una metodología de enseñanza ni de un modelo (en cuanto patrón a seguir), sino de una forma de concebir la ética y su sentido en la configuración del ser psicólogo. La formación en ética profesional, desde esta concepción, enfatizará que el estudiante se apropie de los principios éticos de la profesión, sin por ello dejar de enseñar/aprender los componentes deontológicos y legales involucrados en el ejercicio profesional ético.

\section{Orientaciones para la Formación}

Para una formulación más clara de orientaciones para la formación presentamos a continuación un marco de referencia, en base a un conjunto de consideraciones a tener presente para la toma de decisiones en la materia, para luego proponer procedimientos específicos.

\section{Un Marco de Referencia para la Formación}

Cualquier intento de formular orientaciones de orden educativo tiene que considerar las características de los aprendizajes que se desea lograr, las de los individuos que van a capacitarse y el contexto en que estos aprendizajes se van a aprender y aplicar. En el caso de la formación ética profesional, la revisión realizada nos permite afirmar que nos encontramos frente a un contenido complejo, multidimensional y que abarca a la persona integralmente. Los conocimientos previos, un factor crucial al momento de planificar los aprendizajes, en este caso son el resultado de los procesos de socialización primaria y secundaria. Así, toda la historia personal es depositaria de elementos que tienen la potencialidad de servir de anclaje pero, también, de constituirse en una dificultad, al momento de facilitar la formación en ética profesional.

El estudiante que ingresa a la universidad generalmente lo hace siendo aún adolescente y culminará sus estudios como adulto joven. Esto nos sitúa en un período particular del ciclo vital, sobre el que no es posible afirmar con certeza que la persona ha alcanzado en su desarrollo de juicio moral el nivel postconvencional descrito por Kohlberg (1981), necesario para que la reflexión ética se produzca. 
Perry (1999) además plantea que el desarrollo cognitivo y moral prosigue al menos durante los primeros años universitarios, equivalentes al College estadounidense, y avala su postura con datos de investigación. El autor identifica un esquema de desarrollo a través del cual los jóvenes transitan desde una postura absolutista acerca del conocimiento y los valores hacia una más flexible, en que asumen cierto pluralismo y relativizan sus juicios en función del contexto; para luego entrar en un período en el que experimentan la necesidad de definir y establecer compromisos personales en un mundo que asumen como relativo. El pasaje por la universidad sería, para Perry, un facilitador importante de este proceso de desarrollo.

Una segunda consideración se refiere al impacto en el desarrollo de la identidad que supone la experiencia universitaria. Como viéramos anteriormente, es posible postular que en la universidad los jóvenes experimentarían dos procesos: el apropiarse del ser universitarios y de serlo en una carrera particular. Ambas concepciones suponen énfasis diferentes en la formación en el dominio ético. Una orienta a una formación en valores y preparatoria para asumir roles sociales que se condigan con la condición de ciudadano y profesional con responsabilidades para con la sociedad; la otra se introduce en el campo de la especificidad de la disciplina y de su ejercicio profesional.

Un tercer elemento a tener presente es si se opta por una formación de carácter netamente deontológico o bien por una formación ética en un sentido más amplio. En la primera el foco va a estar en la apropiación del código de ética por parte de los estudiantes; en la segunda se hará un acercamiento de carácter integral a la formación en el dominio éticomoral, en el que una posibilidad es situarse en la perspectiva de la ética positiva.

Finalmente, consideramos esencial tener presentes algunas características del contexto en que hoy en día vivimos y a las cuales hemos aludido anteriormente, tales como el pensamiento posmoderno con la preeminencia del individuo, los desfases entre el desarrollo tecno-científico y los cambios sociales, la emergencia de campos multidisciplinarios en el ámbito profesional y la especialización al interior de las profesiones. Estas condiciones demandan de la formación ética que combine el desarrollo de la autonomía moral con las condiciones para una apropiación exitosa de la identidad profesional.

\section{Procedimientos Especificos}

Ajustándonos al marco de referencia presentado anteriormente, cualquier propuesta para la enseñanza y el aprendizaje del dominio ético debería considerar los distintos momentos del trayecto de la formación, con sus características particulares. Tener presente el poder socializador de la incorporación a la vida universitaria puede ayudar en las tomas de decisiones. Desde esa perspectiva, habrá que buscar los medios para lograr la mayor coherencia posible entre el currículo explícito, en el que se plasmarán la decisiones de corte pedagógico, y el currículo oculto o implícito que los estudiantes aprenden de forma incidental por el solo hecho de participar de una experiencia educacional. Resulta conveniente preguntarse cuál es el sentido de la experiencia universitaria en la institución donde se proyecta esta formación, los valores que la sustentan y, en consecuencia, el tipo de profesional que se está promoviendo.

Sobre esta base, distinguimos entre una fase de formación inicial, desde el ingreso y hasta la licenciatura, y un ciclo profesional, sea este anterior o posterior a la obtención del título profesional, pero en todo caso conducente a la habilitación para el ejercicio profesional. En ambas fases debería contemplarse cómo favorecer la toma de conciencia de problemas éticos y la reflexión en torno a ellos, la facilitación del autoconocimiento en la dimensión ético-moral y la promoción de una actitud reflexiva e introspectiva que favorezca el auto-examen con respecto a esta dimensión.

En el ciclo de formación inicial, la toma de conciencia de problemas éticos puede favorecerse con el tratamiento de los problemas cotidianos de la convivencia en la universidad y el conocimiento de la realidad social. Interiorizarse de la misión de la universidad con respecto a su responsabilidad social y de cómo la ha asumido a lo largo de 
su historia, y discutirla, puede ser un factor sensibilizador adicional y, además, un facilitador de la inserción en la cultura universitaria. Es la oportunidad para considerar un trabajo en torno al ejercicio de la ciudadanía y la eventual implementación de un programa de responsabilidad social universitaria, transversal a todas las carreras de la institución.

En el ámbito específico de la carrera, puede hacerse otro tanto con los principios y valores de la psicología, con el apoyo de la Declaración Universal. Esto puede acompañarse de un primer acercamiento a la deontología de la profesión, con una lectura inicial del Código de Ética de la Orden, pero circunscribiendo la reflexión fundamentalmente en torno a sus principios. Debería propiciarse, también, la reflexión sobre problemas relativos a la ética situada en el ser estudiantes universitarios y, particularmente, a los que emerjan en el devenir cotidiano. Hacia el final de este ciclo, teniendo ya los estudiantes un mayor conocimiento de la carrera, podría inducirse un trabajo de introspección buscando la relación entre la historia y los valores personales y la elección de la carrera de psicología. Puede considerarse, además, el empleo de metodologías de enseñanza que sensibilicen a la realidad social, como es el caso del aprendizaje en servicio (Arratia Figueroa, 2008).

El ciclo profesional sería el momento para conocer a fondo el código de ética profesional y las normativas legales asociadas al ejercicio profesional. La reflexión ética podrá girar en torno a casos concretos y basados en situaciones reales, idealmente con el apoyo de algún modelo de toma de decisiones. Podrá continuar la revisión de la historia personal, aquí con el apoyo de un genograma ético, en la medida en que pueda garantizarse a los estudiantes un espacio cómodo y protegido, dado el riesgo que siempre conlleva el trabajo introspectivo asociado a las figuras significativas como, por ejemplo, las parentales.

En todo trabajo introspectivo, independiente del momento del trayecto formativo en que se efectúe, habrá que asegurar el carácter voluntario de la autorrevelación y buscar mecanismos para garantizar su confidencialidad. La elaboración de portafolios y bitácoras puede constituir un recurso potente para facilitar la introspección.

\section{Reflexiones al Cierre}

En la formación universitaria en ética identificamos dos tendencias, que bien pueden complementarse entre sí. Una deviene del ser universitario, de carácter transversal a toda experiencia universitaria por el solo hecho de serlo, y cuya razón de ser se halla en la responsabilidad del profesional con su sociedad. Es el marco que fundamenta que en la educación universitaria se contemple la inserción de estrategias orientadas a la formación del individuo integralmente como persona y como ciudadano. La otra consiste en la formación en ética profesional, proceso que debería tener su énfasis en el ciclo profesional pero iniciarse desde los primeros años de la carrera, debido a la relevancia de la ética como componente de la identidad profesional.

El desarrollo acelerado del conocimiento en la actualidad proporciona un nuevo perfil a las carreras profesionales. Emergen nuevas tareas, la tecnología proporciona herramientas novedosas para el ejercicio laboral y el trabajo puede significar la incorporación a equipos multidisciplinarios. En este contexto aumentan las dificultades para orientar la actuación profesional en su marco ético, ya que los profesionales deben aplicar la deontología profesional en situaciones para los cuales esta no fue pensada. Surge de aquí la necesidad de facilitar el desarrollo de la autonomía moral y un fuerte arraigo en los principios de la ética profesional, que permita dirimir frente a la complejidad ética de las situaciones que se enfrentan en el ejercicio profesional.

La formación en ética en psicología no se satisface solamente con la enseñanza del código de ética profesional, aunque se complemente con la revisión de teorías filosóficas y eventualmente con el análisis y el debate de situaciones éticamente delicadas del campo profesional. Si no se considera que los "conocimientos previos" descansan en logros del desarrollo ontológico, en la historia personal y en los sistemas de creencias de cada uno, se correrá el riesgo de transformarla en un entrenamiento 
mecánico y con una connotación negativa. Debe orientarse de modo que este elemento constitutivo de la identidad profesional sea comprendido como tal y en una doble orientación, en beneficio del prójimo y, a través suyo, del propio profesional.

En este proceso hay que ayudar a los estudiantes a darse cuenta que las decisiones éticas siempre son, en última instancia, personales y descansan en la conciencia individual. Por eso mismo, corresponde a cada uno asumir las consecuencias de las propias opciones y elecciones y los efectos que estas tienen en el otro.

\section{Referencias}

Ajzen I. (1996). The social psychology of decision making. En E. T. Higgins \& A. W. Kruglanski (Eds.), Social psychology handbook of basic principles (pp. 297-328). New York: Guilford.

Alvear, K., Pasmanik, D., Winkler, M. I. \& Olivares, B. (2008). ¿Códigos en la posmodernidad? Opiniones de psicólogos/as acerca del Código de Ética Profesional del Colegio de Psicólogos de Chile A. G. Terapia Psicológica, 26, 215-228.

Ansell, C. \& Ross, H. L. (1995). Reply to Pope and Bajt. En D. N. Bersoff, Ethical conflicts in psychology $\left(2^{\text {th }}\right.$ ed., p. 101). Washington, DC: American Psychological Association.

Arratia Figueroa, A. (2008). Ética, solidaridad y "aprendizaje servicio" en la educación superior [Versión electrónica]. Acta Bioethica, 14, 61-67.

Bauman, Z. (2004). Ética posmoderna. Buenos Aires: Siglo XXI.

Bilbeny, N. (1997). La revolución en la ética: hábitos $y$ creencias en la sociedad digital. Barcelona: Anagrama.

Blanco, L. \& Ite, A. (1994). Estudio descriptivo sobre la opinión de los psicólogos titulados de universidades del Área Metropolitana acerca de su formación profesional. Tesis no publicada para optar al título de Psicólogo. Pontificia Universidad Católica de Chile, Santiago, Chile.

Bolívar, A. (2005). El lugar de la ética profesional en la formación universitaria. Revista Mexicana de Investigación Educativa, 10, 93-123.

Boni, A. \& Lozano, J. F. (2005). El aprendizaje ético en la universidad y su relación con las competencias transversales en el diseño de los nuevos planes de estudio. Tarragona, España: Universitat Rovira i Virgili. Extraído el 20 Diciembre, 2005, de http:// www.sre.urv.es/web/aulafutura/php/fitxers/364. $\mathrm{pdf}$

Calo, O. (2000). Ética y deontología en la formación del psicólogo argentino. Fundamentos en Humanidades, 2, 7-12.

Colegio de Psicólogos de Chile (1999). Código de ética profesional. Santiago, Chile: Autor.

Cortina, A. (1995). La ética de la sociedad civil. Madrid: Anaya.
De las Fuentes, C., Willmuth, M. E \& Yarrow, C. (2005). Competency training in ethics education and practice. Professional Psychology: Research and Practice, 36, 362-366.

Delors, J. (1996). Informe a la UNESCO de la Comisión Internacional sobre la Educación para el Siglo XXI. Madrid: Santillana.

Díaz, E. (1999). Posmodernidad. Buenos Aires: Biblos.

Falender, C. (2006, Agosto). Ethics in the practicum and internship. Ponencia presentada en la $114^{\text {th }}$ Annual Convention of the American Psychological Association, New Orleans, LA, Estados Unidos. Extraído el 10 Febrero, 2007, de http://www. cfalender.com/apa.pdf

Fine, M. A. \& Lawrence, P. U. (1995). Integrating psychology and philosophy in teaching a graduate course in ethics. En D. N. Bersoff, Ethical conflicts in psychology ( $2^{\text {th }}$ ed., pp. 116-117). Washington, DC: American Psychological Association.

González, J. \& Wagennar, R. (Eds.). (2003). Tuning educational structures in Europe. Informe final fase uno. Bilbao: Universidad de Deusto. Extraído el 17 Febrero, 2009, de http://www.relint. deusto.es/TUNINGProject/spanish/doc_fase1/ Tuning\%20Educational.pdf

González Maura, V. \& González Tirados, R. M. (2008). Competencias genéricas y formación profesional: un análisis desde la docencia universitaria. Revista Iberoamericana de Educación, 47, 185-209.

Gyarmati, G. (1984). Las profesiones: conocimiento y el poder. Santiago, Chile: Ediciones Universidad Católica de Chile.

Handelsman, M.M.(1995). Problems with ethics training by "osmosis". En D. N. Bersoff, Ethical conflicts in psychology (2 ${ }^{\text {th }}$ ed., pp. 110-112). Washington, DC: American Psychological Association.

Handelsman, M. M., Gottlieb, M. C. \& Knapp, S. (2005). Training ethical psychologists: An acculturation model. Professional Psychology: Research and Practice, 36, 59-65.

Hirsch Adler, A. (2003). Elementos significativos de la ética profesional [Versión electrónica]. Reencuentro, 38, 8-15

Holzapfel, C. (2000). Aventura ética, hacia una ética originaria. Santiago, Chile: Universidad de Chile, Facultad de Filosofía y Humanidades.

Kalichman, S. C. (1995). Reporting laws, confidentiality, and clinical judgment: Reply to Ansell and Ross. En D. N. Bersoff, Ethical conflicts in psychology (2 ${ }^{\text {th }}$ ed., p. 116). Washington, DC: American Psychological Association.

Knapp, S. J. \& VandeCreek, L. D. (2006). Practical ethics for psychologists. A positive approach. Washington, DC: American Psychological Association.

Kohlberg, L. (1981). Essays on moral development: The philosophy of moral development. New York: Harper and Row.

Lipovetsky, G. (1998). La era del vacío. Ensayos sobre el individualismo contemporáneo. Barcelona: Anagrama.

Martínez, M., Buxarrais, M. R. \& Bara, F. E. (2002). La universidad como espacio de aprendizaje ético [Versión electrónica]. Revista Iberoamericana de Educación, 29, 17-43. 
Martínez de Carrasquero, C., Mavárez, R. J., Ligibther, A. R. \& Carvallo, B. (2008). La responsabilidad social universitaria como estrategia de vinculación con su entorno social [Versión electrónica]. Frónesis, 15(3), 81-103.

Montero, M. (2004). Introducción a la psicología comunitaria. Buenos Aires: Paidós.

Morales, M., Sziklai, G., Díaz, R. \& Scharager, J. (1988). La formación profesional de los psicólogos en Chile. Análisis de la opinión de psicólogos y estudiantes de la carrera. Revista Chilena de Psicología, 9(1), 57-64.

Orellana Benado, M. E. (1994). Pluralismo: una ética para el siglo XXI. Santiago, Chile: Editorial Universidad de Santiago.

Perry, W. G. (1999). Forms of ethical and intellectual development in the college years. A scheme. San Francisco, CA: Jossey-Bass.

Pope, K. S. \& Bajt, T. R. (1995). When laws and values conflict: A dilemma for psychologists. En D. N. Bersoff, Ethical conflicts in psychology $\left(2^{\text {th }}\right.$ ed., pp. 99-100). Washington, DC: American Psychological Association.

Real Academia Española (2001). Diccionario de la lengua española (22 $2^{\mathrm{a}}$ ed.). Madrid: Autor. Extraído el 14 Enero, 2008, de http://buscon.rae.es/draeI

Fecha de recepción: Marzo de 2009.

Fecha de aceptación: Septiembre de 2009.
Rest, J. (1979). Revised manual for de Defining Issues Test. Minneapolis, MN: University of Minnesota Press.

Rest, J. (1983). Morality. En P. H. Mussen (Ed.), Handbook of child psychology, Vol. 3 (pp. 556629). New York: Wiley.

Rest, J. \& Narváez, D. (1994). Moral development in the professions: Psychology and applied ethics. Hillsdale, NJ: Lawrence Erlbaum Associates.

Sociedad Interamericana de Psicología (2008). Declaración Universal de Principios Éticos para Psicólogas y Psicólogos. San Juan, Puerto Rico: Autor. Extraído el 11 Febrero, 2009, de http://www.sipsych.org/Decla racioUniversaldeprincipiosEticos.pdf

Van Eenwyk, J. R. (1995). When laws and values conflict: Comment on Pope and Bajt. En D. N. Bersoff, Ethical conflicts in psychology ( $2^{\text {th }}$ ed., p. 102). Washington, DC: American Psychological Association.

Vattimo, G. (2004). Nihilismo y emancipación. Ética, política y derecho. Buenos Aires: Paidós.

Villa, A. \& Poblete, M. (2007). Aprendizaje basado en competencias: una propuesta para la evaluación de las competencias genéricas. Bilbao: Ediciones Mensajero. 